\title{
Scalar leptoquark pair production at hadron colliders
}

\author{
Christoph Borschensky $\odot,{ }^{1, *}$ Benjamin Fuks $\odot,{ }^{2,3, \uparrow}$ Anna Kulesza, ${ }^{4, *}$ and Daniel Schwartländer ${ }^{4, \S}$ \\ ${ }^{1}$ Institute for Theoretical Physics, University of Tübingen, Auf der Morgenstelle 14, \\ 72076 Tübingen, Germany \\ ${ }^{2}$ Sorbonne Université, CNRS, Laboratoire de Physique Théorique et Hautes Énergies, \\ LPTHE, F-75005 Paris, France \\ ${ }^{3}$ Institut Universitaire de France, 103 boulevard Saint-Michel, 75005 Paris, France \\ ${ }^{4}$ Institute for Theoretical Physics, WWU Münster, D-48149 Münster, Germany
}

(Received 4 March 2020; accepted 2 June 2020; published 16 June 2020)

\begin{abstract}
We revisit scalar leptoquark pair production at hadron colliders. Apart from QCD contributions, we include the lepton $t$-channel exchange diagrams relevant in the light of the recent $B$-flavor anomalies. We evaluate all contributions at next-to-leading order in QCD and resum, in the threshold regime, soft-gluon radiation at next-to-next-to-leading-logarithmic accuracy. All corrections are found equally relevant. Our predictions consist of the most precise leptoquark cross section calculations available to date and are necessary for the best exploitation of leptoquark LHC searches.
\end{abstract}

DOI: 10.1103/PhysRevD.101.115017

\section{INTRODUCTION}

Many extensions of the Standard Model (SM) predict the existence of scalar leptoquarks [1-8], i.e., scalar bosons coupling to a quark and a lepton simultaneously. Evidence for their existence is consequently vastly searched for at the LHC. However, none of the recent ATLAS $[9,10]$ and CMS [11-15] analyses find any hint for these leptoquarks, so that their mass is now constrained to be larger than $1-1.5 \mathrm{TeV}$. Recently, scalar leptoquarks have gained significant interest as they may provide an explanation [16-22] for the $B$-meson anomalies [23-30] and address [31] the discrepancy between theoretical predictions [32] and experimental measurements [33] of the anomalous magnetic moment of the muon $(g-2)_{\mu}$. In this context, favored scenarios generally feature large lepton-quark-leptoquark Yukawa couplings $y$.

The most stringent bounds originating from LHC direct searches for leptoquark pair production and decay are extracted by assuming that leptoquarks are solely produced via strong interactions. In other words, non-QCD diagrams involving lepton $t$-channel exchanges of $\mathcal{O}\left(y^{2}\right)$ are neglected. In the associated limit setting procedure, signal cross sections evaluated at next-to-leading-order (NLO)

\footnotetext{
*christoph.borschensky@uni-tuebingen.de

fuks@1pthe.jussieu.fr

*anna.kulesza@uni-muenster.de

§d_schw20@uni-muenster.de
}

Published by the American Physical Society under the terms of the Creative Commons Attribution 4.0 International license. Further distribution of this work must maintain attribution to the author(s) and the published article's title, journal citation, and DOI. Funded by SCOAP. accuracy in the strong coupling $\alpha_{s}$ [11-15], sometimes also supplemented by logarithmic threshold corrections $[9,10]$, are used. Thus the predictions include contributions at $\mathcal{O}\left(\alpha_{s}^{2}\right)$ and $\mathcal{O}\left(\alpha_{s}^{3}\right)$, or possibly of higher order in $\alpha_{s}$, but are independent of $y[34,35]$. Bearing in mind the $B$-anomalies and $(g-2)_{\mu}$ motivation, the limits may thus be incorrectly estimated.

In this paper, we perform for the first time a full NLO-QCD cross section calculation for scalar leptoquark pair production at hadron colliders, in which we include both the QCD and $t$-channel contributions. Hadronic production of heavy systems, which is the case considered here, inevitably probes partonic center-of-mass energies close to the production threshold given by twice the leptoquark mass $m_{\mathrm{LQ}}$. In this limit, radiative corrections are dominated by soft-gluon emissions, manifesting themselves as large logarithmic terms that must be consistently resummed to all orders [36-39]. We report here threshold-resummed results at next-to-next-toleading-logarithmic (NNLL) accuracy and showcase predictions obtained by matching them to our new NLO results. In the following, we first present the considered theoretical framework and provide brief technical computational details. We then show an illustrative selection of results that underlines how all considered corrections affect the results in comparable and significant ways. Our predictions, which are the most precise to date, are hence required to derive limits consistently, in particular when assessing the influence of the leptoquark Yukawa couplings.

\section{THEORETICAL FRAMEWORK}

We focus on a simplified model in which the SM is supplemented by several species of scalar leptoquarks 
$S_{1}, \tilde{S}_{1}, R_{2}, \tilde{R}_{2}$ and $S_{3}$. Inspired by standard naming conventions [40,41], these leptoquarks lie in the $(\mathbf{3}, \mathbf{1})_{-1 / 3}$, $(\mathbf{3}, \mathbf{1})_{-4 / 3},(\mathbf{3}, \mathbf{2})_{7 / 6},(\mathbf{3}, \mathbf{2})_{1 / 6}$ and $(\mathbf{3}, \mathbf{3})_{-1 / 3}$ representations of the SM gauge group respectively, and we target their Yukawa interactions involving exactly one lepton and quark. The latter are collected in the Lagrangian:

$$
\begin{aligned}
\mathcal{L}_{\text {int }}= & \mathbf{y}_{\mathbf{1}}^{\mathbf{R R}} \bar{u}_{R}^{c} \ell_{R} S_{1}^{\dagger}+\mathbf{y}_{\mathbf{1}}^{\mathbf{L L}}\left(\bar{Q}_{L}^{c} \cdot L_{L}\right) S_{1}^{\dagger}+\tilde{\mathbf{y}}_{\mathbf{1}}^{\mathbf{R R}} \bar{d}_{R}^{c} \ell_{R} \tilde{S}_{1}^{\dagger} \\
& +\mathbf{y}_{2}^{\mathbf{L R}} \bar{e}_{R} Q_{L} R_{2}^{\dagger}+\mathbf{y}_{\mathbf{2}}^{\mathbf{R L}} \bar{u}_{R}\left(L_{L} \cdot R_{2}\right)+\tilde{\mathbf{y}}_{\mathbf{2}}^{\mathbf{R L}} \bar{d}_{R}\left(L_{L} \cdot \tilde{R}_{2}\right) \\
& +\mathbf{y}_{\mathbf{3}}^{\mathbf{L L}}\left(\bar{Q}_{L}^{c} \cdot \sigma_{k} L_{L}\right)\left(S_{3}^{k}\right)^{\dagger}+\text { H.c. }
\end{aligned}
$$

In this expression, all flavor indices are suppressed for clarity, $\sigma_{k}$ stands for the Pauli matrices and the dot for the invariant product of two fields lying in the (anti)fundamental representation of $S U(2)$. The $Q_{L}$ and $L_{L}$ spinors denote the SM weak doublets of left-handed quarks and leptons, and $u_{R}, d_{R}$ and $\ell_{R}$ are the corresponding weak singlets. Moreover, the $\mathbf{y} / \tilde{\mathbf{y}}$ couplings are $3 \times 3$ matrices in the flavor space, the first index of any element $y_{i j} / \tilde{y}_{i j}$ referring to the quark generation and the second one to the lepton generation in the gauge basis.

The calculations reported in this work concern scalar leptoquark pair production and include fixed order contributions at leading order (LO) and NLO in QCD. In contrast with previous work $[34,35,42,43]$, we not only consider the QCD components at $\mathcal{O}\left(\alpha_{s}^{2}\right)$ and $\mathcal{O}\left(\alpha_{s}^{3}\right)$, but also include the $t$-channel lepton exchange contributions at $\mathcal{O}\left(y^{4}\right)$ and $\mathcal{O}\left(y^{4} \alpha_{s}\right)$ as well as the $\mathcal{O}\left(y^{2} \alpha_{s}\right)$ and $\mathcal{O}\left(y^{2} \alpha_{s}^{2}\right)$ interference of the $t$-channel diagrams with the QCD ones. The full NLO-accurate predictions are collectively coined "NLO w/ $t$-channel" in the following, in contrast to the pure QCD ones that we refer to as the "NLO-QCD" predictions. The NLO $\mathrm{w} / t$-channel cross sections are then additively matched with the resummed NNLL soft-gluon contributions, resulting in cross section predictions at NLO $\mathrm{w} / t$-channel+NNLL accuracy. Threshold resummation is performed in Mellin space (see e.g., [39]) and involves one-loop matching coefficients [44].

To ensure the correctness of the results, we perform the calculations in two independent ways: We first implement the above model into FeynRules [45], which we jointly use with NLOCT [46] and FeynArts [47] to renormalize the bare Lagrangian of Eq. (1) at $\mathcal{O}\left(\alpha_{s}\right)$. We then generate a UFO model file [48] that we use to evaluate fixed-order LO and NLO predictions within the MG5_aMC framework [49]. The latter are cross-checked with results obtained within the POWHEG-BOX framework [50], in which we input virtual corrections calculated with the FeynArts, FormCalc [51] and COLLIER [52-55] packages. The NNLL corrections are evaluated with two independent in-house Monte Carlo codes.

\section{SCALAR LEPTOQUARK PAIR PRODUCTION AT THE LHC}

We present selected predictions for scalar leptoquark pair production at the $13 \mathrm{TeV}$ LHC for the three most commonly discussed types of scalar leptoquarks in the context of the flavor anomalies: the $S U(2)_{L}$ singlet state $S_{1}$ (denoted by $S_{1}^{(-1 / 3)}$ due to its electric charge of $\left.-1 / 3\right)$, doublet state $R_{2}$ and the triplet state $S_{3}$. More specifically, in the last two cases, we consider the pair production of the $R_{2}$ mass eigenstate of electric charge of $5 / 3$ (denoted by $R_{2}^{(5 / 3)}$ ) and the one of the $S_{3}$ mass eigenstate of electric charge of $-4 / 3$ (denoted by $\left.S_{3}^{(-4 / 3)}\right)$. In all our calculations, we treat the leptoquark mass $m_{\mathrm{LQ}}$ as a free parameter and assume the Cabibbo-KobayashiMaskawa matrix (CKM) matrix to be diagonal. While the determination of a scenario compatible with flavor constraints and $Z$-pole observables is desirable [56], this goes beyond the scope of this study. We consider instead benchmarks motivated by Ref. [21]. The values of the Yukawa couplings found in this study were obtained in a fit to low-energy observables and did not involve constraints from direct searches for leptoquarks at the LHC. Given that the description of lepton flavor university-violating observables involves both leptoquark couplings and masses, optimally one should aim at a global fit based on direct and indirect constraints, in which case the calculations presented in this work will play a crucial role. For $S_{1} S_{1}^{*}$ production, we adopt a minimal flavor ansatz for the leptoquark Yukawa couplings, $\left(\mathbf{y}_{1}^{\mathbf{L L}}\right)_{22}=-0.15$ and $\left(\mathbf{y}_{1}^{\mathbf{L L}}\right)_{32}=3$ with all other $\mathbf{y}_{\mathbf{1}}^{\mathbf{L L}}$ elements set to 0 . For $R_{2} R_{2}^{*}$ production, we similarly consider as the only nonvanishing coupling $\left(\mathbf{y}_{\mathbf{2}}^{\mathbf{R L}}\right)_{22}=1.5$, a value still allowed by direct exclusion bounds [21], while for $S_{3} S_{3}^{*}$ production, we adopt $\left(\mathbf{y}_{\mathbf{3}}^{\mathbf{L L}}\right)_{22}=-\left(\mathbf{y}_{\mathbf{3}}^{\mathbf{L L}}\right)_{32}$, keeping the actual coupling value free and setting all other couplings to 0 .

Our results are obtained by convoluting the partonic results with two different sets of parton distribution functions (PDFs), NNPDF3.1 [57] and CT18 [58]. Unless stated otherwise, NLO sets are employed for NLO-QCD and NLO $w / t$-channel predictions, while NNLO sets are used for NLO + NNLL calculations. We set the renormalization $\left(\mu_{R}\right)$ and factorization $\left(\mu_{F}\right)$ scales equal to a common value $\mu=\mu_{R}=\mu_{F}$. The central scale choice $\mu=\mu_{0}$ is fixed to $\mu_{0}=m_{\mathrm{LQ}}$, and scale uncertainties are estimated by varying $\mu$ by a factor of 2 up and down.

In Fig. 1, we present cross section predictions for $S_{1}^{(-1 / 3)} S_{1}^{(1 / 3)}$ (left column) and $R_{2}^{(5 / 3)} R_{2}^{(-5 / 3)}$ (right column) production, both for the NNPDF3.1 (upper row) and CT18 (lower row) parton densities. We estimate the relative importance of the various corrections studied in this work with respect to NLO-QCD predictions, and assess the size of the scale and PDF uncertainties. Comparing the four subfigures, we observe that depending on the process, the PDFs, the magnitude of the Yukawa couplings, and $m_{\mathrm{LQ}}$, the considered corrections can influence the predictions in different, often contrasting, ways. 

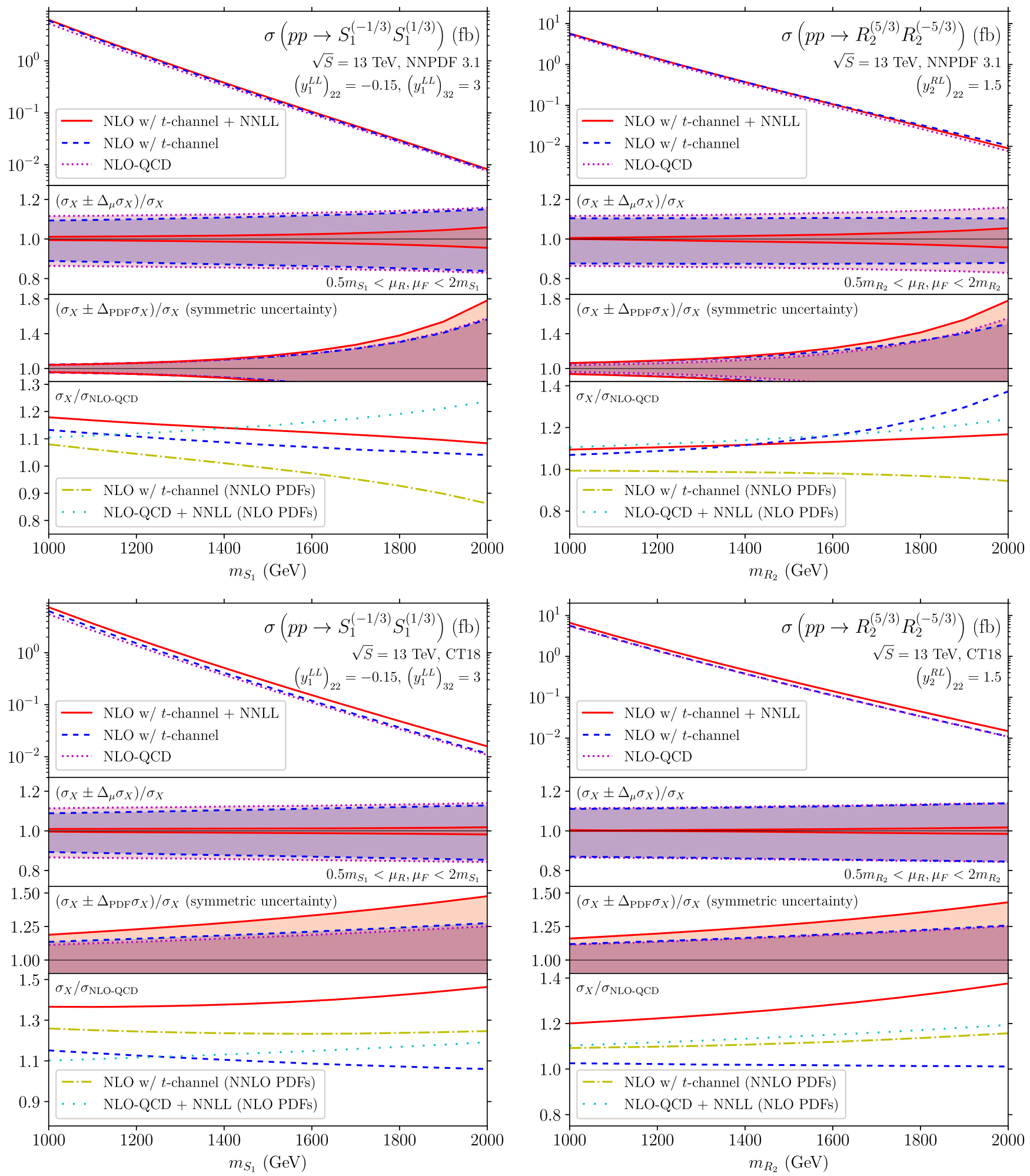

FIG. 1. $S_{1}^{(-1 / 3)} S_{1}^{(1 / 3)}$ (left) and $R_{2}^{(5 / 3)} R_{2}^{(-5 / 3)}$ (right) production at the $13 \mathrm{TeV}$ LHC, using the NNPDF3.1 (upper row) and CT18 (lower row) PDF sets. In the top panels of the subfigures, we present cross section predictions at the NLO-QCD (magenta dotted), NLO $\mathrm{w} / t$-channel (blue dashed) and NLO $\mathrm{w} / t$-channel+NNLL (red solid) accuracy. The associated scale and PDF uncertainties are also displayed (middle panels). In the lower panels, we show ratios of the NLO w/t-channel, NLO w/t-channel+NNLL, NLO $\mathrm{w} / t$-channel calculated using NNLO PDFs (olive dash-dotted) and NLO-QCD + NNLL (turquoise dotted) results to the NLO-QCD cross section. 

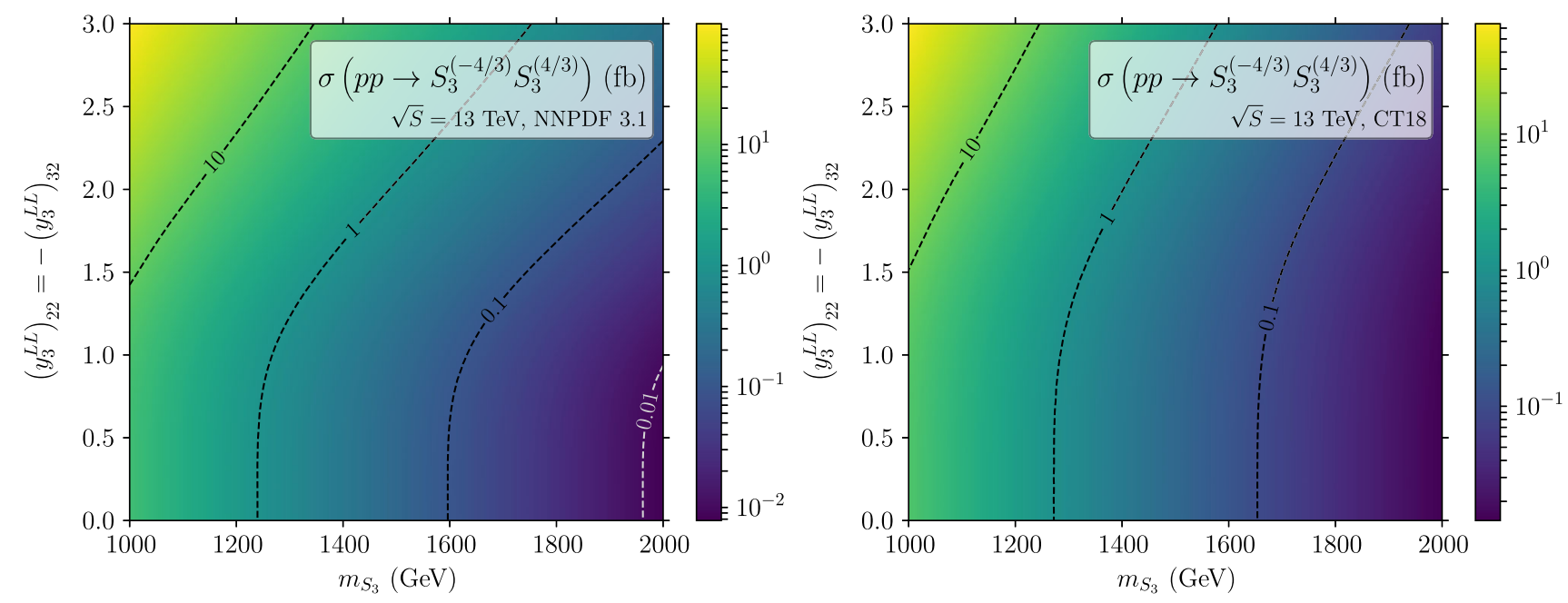

FIG. 2. NLO w/t-channel+NNLL total cross section for $S_{3}^{(-4 / 3)} S_{3}^{(4 / 3)}$ production at the $13 \mathrm{TeV}$ LHC as a function of the $S_{3}$ mass $m_{\mathrm{LQ}}=m_{S_{3}}$ and the Yukawa couplings $\left(\mathbf{y}_{\mathbf{3}}^{\mathbf{L L}}\right)_{22}=-\left(\mathbf{y}_{\mathbf{3}}^{\mathbf{L L}}\right)_{32}$ (all other Yukawa couplings being set to 0 ). We present predictions obtained with the NNPDF3.1 (left) and CT18 (right) PDF set.

Although providing a positive correction, the size of the $t$-channel contributions depends very differently on $m_{\mathrm{LQ}}$ for the two processes (blue dashed curves). On the contrary, NNLL effects, which we estimate through the ratio of the NLO-QCD + NNLL to the NLO-QCD cross sections both calculated with the same NLO PDF set (turquoise dotted curves), are independent of the process and PDF choice. As expected, this ratio is bigger than 1 for all $m_{\mathrm{LQ}}$ considered, and grows with increasing $m_{\mathrm{LQ}}$, i.e., approaching the production threshold.

This behavior is vastly modified by the interplay of $t$-channel contributions, PDF effects and soft-gluon corrections, all entering the NNLL results matched with NLO $\mathrm{w} / t$-channel (red solid curves). The effect of evaluating the NLO w/ $t$-channel cross sections with NNLO PDF sets instead of NLO sets is illustrated by the difference between the corresponding ratios to NLO QCD predictions (blue dashed vs olive dash-dotted curves). For the NNPDF3.1 PDF set (upper row), this effect diminishes the cross sections, offsetting the increase stemming from the NNLL contributions. As a consequence, the NLO w/tchannel+NNLL results deliver a positive correction of about $10 \%-20 \%$ with respect to the NLO-QCD predictions for $m_{\mathrm{LQ}} \in[1,2] \mathrm{TeV}$. Similarly for the NLO $\mathrm{w} / t$-channel result, the full NLO $\mathrm{w} / t$-channel+NNLL correction exhibits the opposite behavior with increasing $m_{\mathrm{LQ}}$ in the $S_{1}$ (upper left) and $R_{2}$ (upper right) cases.

When CT18 PDFs are used instead (lower row), the corrections are larger and reach a magnitude of about $20 \%-50 \%$, the impact this time increasing with $m_{\mathrm{LQ}}$ for both processes. Furthermore, in the NNPDF3.1 case, various contributions to the total correction are often much bigger than the correction itself. For example, the correction due to including $t$-channel diagrams reaches up to
$40 \%$ of the NLO-QCD result for the pair production of $2 \mathrm{TeV} R_{2}$ leptoquarks, whereas the complete NLO $\mathrm{w} / t$-channel+NNLL one is only of about $20 \%$. In contrast, results obtained with CT18 densities exhibit the opposite behavior, the cross sections being typically enhanced when switching from NLO to NNLO PDFs. The $t$-channel and soft-gluon resummation pieces are of comparable size and thus equally contribute to the combined correction. Therefore a precise knowledge of the cross section requires calculating all classes of corrections.

In the middle panels of the four subfigures of Fig. 1, we focus on scale and PDF uncertainties. We distinguish the impact of scale variations (second panel) from the one originating from the PDF determination (third panel). Our results show that soft-gluon resummation leads to a significant reduction of the scale uncertainties from around $10 \%$ (for the NLO predictions) to about $1 \%-2 \%$ for $m_{\mathrm{LQ}}$ values ranging up to slightly above the current exclusion limits. The reduction might however be underestimated due to the chosen method for scale uncertainty evaluation. This calls for a more comprehensive study. Correspondingly, the total theoretical error for our final NLO w $/ t$-channel $+\mathrm{NNLL}$ predictions is dominated by its PDF component. The size of the PDF error is however strongly dependent on the PDF choice. For instance, results derived with NNPDF3.1 exhibit, for $m_{\mathrm{LQ}} \sim 1 \mathrm{TeV}$, PDF errors smaller or comparable in magnitude to the size of the perturbative corrections, whilst at higher $m_{\mathrm{LQ}}$ values, the PDF error becomes significantly bigger. In comparison, PDF errors obtained with the CT18 set are larger for small $m_{\mathrm{LQ}}$ values, but do not grow as quickly for higher masses. Still, the PDF errors turn out to be of the same order as the full perturbative corrections for large $m_{\mathrm{LQ}}$ values. Those large PDF errors at high masses hence obscure the accuracy of the predictions. However, as more LHC data 
will be analyzed, one can expect a substantial improvement of the PDF knowledge, in particular in the large Bjorken- $x$ regime, so that the PDF errors associated with predictions relevant for high-mass system production will be significantly reduced.

In Fig. 2, we calculate the NLO w $/ t$-channel+NNLL total cross section for $S_{3}^{(-4 / 3)} S_{3}^{(4 / 3)}$ production, and study its dependence on the leptoquark mass and Yukawa coupling strength. We consider both the NNPDF3.1 (left) and CT18 (right) PDF sets. At small values of the Yukawa coupling, the dominant production mechanism is QCD driven so that the cross section solely depends on $m_{\mathrm{LQ}}$. On the contrary, as the coupling approaches 1 , the $t$-channel contributions become more relevant and the total rate significantly increases. This behavior is mostly independent of the chosen PDF set, CT18 predictions being slightly less sensitive to the $t$-channel diagrams.

\section{SUMMARY}

We have significantly advanced the precision of scalar leptoquark pair production cross section computations. First, we have included all contributions to the process, both the QCD ones and those involving the $t$-channel exchange of a lepton, at NLO QCD. Second, we have resummed soft-gluon radiation in the threshold regime to NNLL accuracy.

The $t$-channel contributions, threshold resummation, the adopted parton densities and benchmark scenario (in particular when the leptoquark Yukawa couplings are taken as large as suggested by the recent $B$-anomalies) importantly affect the total rates, in potentially contrasting and sizable ways. This emphasizes the necessity of including all contributions whose calculation has been pioneered in this work. While the perturbative series exhibits smaller scale uncertainties, the precision of the predictions is limited by the poor PDF knowledge in the large Bjorken- $x$ regime relevant for the production of high-mass systems. In light of our findings, we recommend the usage of NLO w/tchannel+NNLL cross sections, to be taken together with the correspondingly reduced scale uncertainties and PDF errors extracted from the envelope spanned by computations, left for future work, performed with different PDF sets. This follows the strategy outlined in various recommendations for LHC cross section calculations [59-63]. The computer codes used in this work are available upon request.

\section{ACKNOWLEDGMENTS}

We are grateful to V. Hirschi, O. Mattelear and H. S. Shao for their help with MG5_aMC technical issues related to mixed-order computations, as well as to $\mathrm{M}$. Krämer for insightful discussions, and D. Bečirević, D. Guadagnoli and R. Ruiz for useful comments on the manuscript. This work has been supported in part by the DFG Grant No. KU3103/2. We furthermore acknowledge support by the state of Baden-Württemberg through bwHPC and the DFG Grant No. INST 39/963-1 FUGG (bwForCluster NEMO).
[1] J. C. Pati and A. Salam, Lepton number as the fourth color, Phys. Rev. D 10, 275 (1974); Erratum, Phys. Rev. D 11, 703 (1975).

[2] H. Georgi and S. L. Glashow, Unity of All Elementary Particle Forces, Phys. Rev. Lett. 32, 438 (1974).

[3] H. Fritzsch and P. Minkowski, Unified interactions of leptons and hadrons, Ann. Phys. (N.Y.) 93, 193 (1975).

[4] S. Dimopoulos and L. Susskind, Mass without scalars, Nucl. Phys. B155, 237 (1979); 2, 930 (1979).

[5] G. Senjanovic and A. Sokorac, Light leptoquarks in SO(10), Z. Phys. C 20, 255 (1983).

[6] B. Schrempp and F. Schrempp, Light leptoquarks, Phys. Lett. 153B, 101 (1985).

[7] J. L. Hewett and T. G. Rizzo, Low-energy phenomenology of superstring inspired E(6) models, Phys. Rep. 183, 193 (1989).

[8] P. H. Frampton and B.-H. Lee, SU(15) Grand Unification, Phys. Rev. Lett. 64, 619 (1990).

[9] M. Aaboud et al. (ATLAS Collaboration), Searches for scalar leptoquarks and differential cross-section measurements in dilepton-dijet events in proton-proton collisions at a center-of-mass energy of $\sqrt{s}=13 \mathrm{TeV}$ with the ATLAS experiment, Eur. Phys. J. C 79, 733 (2019).

[10] M. Aaboud et al. (ATLAS Collaboration), Searches for third-generation scalar leptoquarks in $\sqrt{s}=13 \mathrm{TeV}$ pp collisions with the ATLAS detector, J. High Energy Phys. 06 (2019) 144.

[11] A. M. Sirunyan et al. (CMS Collaboration), Search for a singly produced third-generation scalar leptoquark decaying to a $\tau$ lepton and a bottom quark in proton-proton collisions at $\sqrt{s}=13 \mathrm{TeV}$, J. High Energy Phys. 07 (2018) 115.

[12] A. M. Sirunyan et al. (CMS Collaboration), Search for pair production of second-generation leptoquarks at $\sqrt{s}=13$ TeV, Phys. Rev. D 99, 032014 (2019).

[13] A. M. Sirunyan et al. (CMS Collaboration), Search for Leptoquarks Coupled to Third-Generation Quarks in Proton-Proton Collisions at $\sqrt{s}=13 \mathrm{TeV}$, Phys. Rev. Lett. 121, 241802 (2018).

[14] A. M. Sirunyan et al. (CMS Collaboration), Search for heavy neutrinos and third-generation leptoquarks in hadronic states of two $\tau$ leptons and two jets in proton-proton collisions at $\sqrt{s}=13 \mathrm{TeV}$, J. High Energy Phys. 03 (2019) 170. 
[15] A. M. Sirunyan et al. (CMS Collaboration), Search for pair production of first-generation scalar leptoquarks at $\sqrt{s}=13$ TeV, Phys. Rev. D 99, 052002 (2019).

[16] M. Bauer and M. Neubert, Minimal Leptoquark Explanation for the $\mathrm{R}_{D^{(*)}}, \mathrm{R}_{K}$, and $(g-2)_{g}$ Anomalies, Phys. Rev. Lett. 116, 141802 (2016).

[17] G. Hiller and M. Schmaltz, $R_{K}$ and future $b \rightarrow s \ell \ell$ physics beyond the standard model opportunities, Phys. Rev. D 90, 054014 (2014).

[18] D. Bečirević and O. Sumensari, A leptoquark model to accommodate $R_{K}^{\exp }<R_{K}^{\mathrm{SM}}$ and $R_{K^{*}}^{\exp }<R_{K^{*}}^{\mathrm{SM}}$, J. High Energy Phys. 08 (2017) 104.

[19] A. Crivellin, D. Müller, and T. Ota, Simultaneous explanation of $R\left(D^{(*)}\right)$ and $b \rightarrow s \mu^{+} \mu^{-}$: The last scalar leptoquarks standing, J. High Energy Phys. 09 (2017) 040.

[20] D. Buttazzo, A. Greljo, G. Isidori, and D. Marzocca, B-physics anomalies: A guide to combined explanations, J. High Energy Phys. 11 (2017) 044.

[21] A. Angelescu, D. Bečirević, D. A. Faroughy, and O. Sumensari, Closing the window on single leptoquark solutions to the $B$-physics anomalies, J. High Energy Phys. 10 (2018) 183.

[22] A. Crivellin, D. Müller, and F. Saturnino, Flavor phenomenology of the leptoquark singlet-triplet model, arXiv: 1912.04224.

[23] J. P. Lees et al. (BABAR Collaboration), Evidence for an Excess of $\bar{B} \rightarrow D^{(*)} \tau^{-} \bar{\nu}_{\tau}$ Decays, Phys. Rev. Lett. 109, 101802 (2012).

[24] S. Hirose et al. (Belle Collaboration), Measurement of the $\tau$ Lepton Polarization and $R\left(D^{*}\right)$ in the Decay $\bar{B} \rightarrow D^{*} \tau^{-} \bar{\nu}_{\tau}$, Phys. Rev. Lett. 118, 211801 (2017).

[25] A. Abdesselam et al. (Belle Collaboration), Test of lepton flavor universality in $B \rightarrow K^{*} \ell^{+} \ell^{-}$decays at Belle, arXiv: 1904.02440.

[26] A. Abdesselam et al. (Belle Collaboration), Test of lepton flavor universality in $B \rightarrow K \ell^{+} \ell^{-}$decays, arXiv:1908 .01848 .

[27] R. Aaij et al. (LHCb Collaboration), Measurement of the Ratio of Branching Fractions $\mathcal{B}\left(\bar{B}^{0} \rightarrow D^{*+} \tau^{-} \bar{\nu}_{\tau}\right) /$ $\mathcal{B}\left(\bar{B}^{0} \rightarrow D^{*+} \mu^{-} \bar{\nu}_{\mu}\right)$, Phys. Rev. Lett. 115, 111803 (2015); Erratum, Phys. Rev. Lett. 115, 159901 (2015).

[28] R. Aaij et al. (LHCb Collaboration), Test of lepton universality with $B^{0} \rightarrow K^{* 0} \ell^{+} \ell^{-}$decays, J. High Energy Phys. 08 (2017) 055,

[29] R. Aaij et al. (LHCb Collaboration), Measurement of the ratio of the $B^{0} \rightarrow D^{*-} \tau^{+} \nu_{\tau}$ and $B^{0} \rightarrow D^{*-} \mu^{+} \nu_{\mu}$ Branching Fractions Using Three-Prong $\tau$-Lepton Decays, Phys. Rev. Lett. 120, 171802 (2018).

[30] R. Aaij et al. (LHCb Collaboration), Search for LeptonUniversality Violation in $B^{+} \rightarrow K^{+} \ell^{+} \ell^{-}$Decays, Phys. Rev. Lett. 122, 191801 (2019).

[31] I. Doršner, S. Fajfer, and O. Sumensari, Muon $g-2$ and scalar leptoquark mixing, arXiv:1910.03877.

[32] F. Jegerlehner and A. Nyffeler, The muon $g-2$, Phys. Rep. 477, 1 (2009).

[33] G. W. Bennett et al. (Muon $g-2$ Collaboration), Final report of the muon E821 anomalous magnetic moment measurement at BNL, Phys. Rev. D 73, 072003 (2006).
[34] M. Kramer, T. Plehn, M. Spira, and P. M. Zerwas, Pair production of scalar leptoquarks at the CERN LHC, Phys. Rev. D 71, 057503 (2005).

[35] T. Mandal, S. Mitra, and S. Seth, Pair production of scalar leptoquarks at the LHC to NLO parton shower accuracy, Phys. Rev. D 93, 035018 (2016).

[36] G. F. Sterman, Summation of large corrections to short distance hadronic cross sections, Nucl. Phys. B281, 310 (1987).

[37] S. Catani and L. Trentadue, Resummation of the QCD perturbative series for hard processes, Nucl. Phys. B327, 323 (1989).

[38] H. Contopanagos, E. Laenen, and G. F. Sterman, Sudakov factorization and resummation, Nucl. Phys. B484, 303 (1997).

[39] S. Catani, M. L. Mangano, P. Nason, and L. Trentadue, The resummation of soft gluons in hadronic collisions, Nucl. Phys. B478, 273 (1996).

[40] W. Buchmuller, R. Ruckl, and D. Wyler, Leptoquarks in lepton-quark collisions, Phys. Lett. B 191, 442 (1987); Erratum, Phys. Lett. B 448, 320 (1999).

[41] I. Doršner, S. Fajfer, A. Greljo, J. F. Kamenik, and N. Košnik, Physics of leptoquarks in precision experiments and at particle colliders, Phys. Rep. 641, 1 (2016).

[42] M. Kramer, T. Plehn, M. Spira, and P. M. Zerwas, Pair Production of Scalar Leptoquarks at the Tevatron, Phys. Rev. Lett. 79, 341 (1997).

[43] I. Doršner and A. Greljo, Leptoquark toolbox for precision collider studies, J. High Energy Phys. 05 (2018) 126.

[44] W. Beenakker, C. Borschensky, R. Heger, M. Krämer, A. Kulesza, and E. Laenen, NNLL resummation for stop pair production at the LHC, J. High Energy Phys. 05 (2016) 153.

[45] A. Alloul, N. D. Christensen, C. Degrande, C. Duhr, and B. Fuks, FeynRules 2.0-A complete toolbox for tree-level phenomenology, Comput. Phys. Commun. 185, 2250 (2014).

[46] C. Degrande, Automatic evaluation of UV and R2 terms for beyond the Standard Model Lagrangians: A proof-ofprinciple, Comput. Phys. Commun. 197, 239 (2015).

[47] T. Hahn, Generating Feynman diagrams and amplitudes with FeynRules 3, Comput. Phys. Commun. 140, 418 (2001).

[48] C. Degrande, C. Duhr, B. Fuks, D. Grellscheid, O. Mattelaer, and T. Reiter, UFO_-The universal FeynRules output, Comput. Phys. Commun. 183, 1201 (2012).

[49] J. Alwall, R. Frederix, S. Frixione, V. Hirschi, F. Maltoni, O. Mattelaer, H. S. Shao, T. Stelzer, P. Torrielli, and M. Zaro, The automated computation of tree-level and next-toleading order differential cross sections, and their matching to parton shower simulations, J. High Energy Phys. 07 (2014) 079.

[50] S. Alioli, P. Nason, C. Oleari, and E. Re, A general framework for implementing NLO calculations in shower Monte Carlo programs: The POWHEG BOX, J. High Energy Phys. 06 (2010) 043.

[51] T. Hahn and M. Perez-Victoria, Automatized one loop calculations in four-dimensions and D-dimensions, Comput. Phys. Commun. 118, 153 (1999). 
[52] A. Denner, S. Dittmaier, and L. Hofer, Collier: A Fortranbased complex one-loop library in extended regularizations, Comput. Phys. Commun. 212, 220 (2017).

[53] A. Denner and S. Dittmaier, Reduction of one loop tensor five point integrals, Nucl. Phys. B658, 175 (2003).

[54] A. Denner and S. Dittmaier, Reduction schemes for oneloop tensor integrals, Nucl. Phys. B734, 62 (2006).

[55] A. Denner and S. Dittmaier, Scalar one-loop 4-point integrals, Nucl. Phys. B844, 199 (2011).

[56] P. Arnan, D. Becirevic, F. Mescia, and O. Sumensari, Probing low energy scalar leptoquarks by the leptonic $w$ and $z$ couplings, J. High Energy Phys. 02 (2019) 109.

[57] R. Abdul Khalek et al. (NNPDF Collaboration), A first determination of parton distributions with theoretical uncertainties, Eur. Phys. J. C 79, 838 (2019).

[58] T.-J. Hou et al., New CTEQ global analysis of quantum chromodynamics with high-precision data from the LHC, arXiv:1912.10053.
[59] M. Botje et al., The PDF4LHC working group interim recommendations, arXiv:1101.0538.

[60] S. Dittmaier et al. (LHC Higgs Cross Section Working Group Collaboration), Handbook of LHC Higgs cross sections: 1. Inclusive observables, arXiv:1101.0593.

[61] M. Kramer, A. Kulesza, R. van der Leeuw, M. Mangano, S. Padhi, T. Plehn, and X. Portell, Supersymmetry production cross sections in $p p$ collisions at $\sqrt{s}=7 \mathrm{TeV}$, arXiv: 1206.2892 .

[62] B. Fuks, M. Klasen, D. R. Lamprea, and M. Rothering, Precision predictions for electroweak superpartner production at hadron colliders with Resummino, Eur. Phys. J. C 73, 2480 (2013).

[63] C. Borschensky, M. Krämer, A. Kulesza, M. Mangano, S. Padhi, T. Plehn, and X. Portell, Squark and gluino production cross sections in pp collisions at $\sqrt{s}=13,14,33$ and 100 TeV, Eur. Phys. J. C 74, 3174 (2014). 